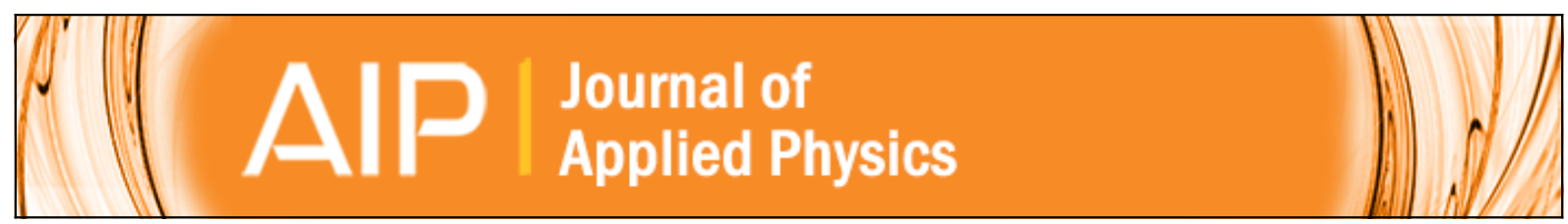

Sub-bandgap absorption in Ti implanted Si over the Mott limit

J. Olea, A. del Prado, D. Pastor, I. Mártil, and G. González-Díaz

Citation: Journal of Applied Physics 109, 113541 (2011); doi: 10.1063/1.3596525

View online: http://dx.doi.org/10.1063/1.3596525

View Table of Contents: http://scitation.aip.org/content/aip/journal/jap/109/11?ver=pdfcov

Published by the AIP Publishing

AAIP Re-register for Table of Content Alerts

Create a profile.

Sign up today! 


\title{
Sub-bandgap absorption in Ti implanted Si over the Mott limit
}

\author{
J. Olea, A. del Prado, D. Pastor, ${ }^{\text {a) }}$ I. Mártil, and G. González-Díaz \\ Dpto. Física Aplicada III (Electricidad y Electrónica), Facultad de Ciencias Físicas, \\ University Complutense, 28040 Madrid, Spain
}

(Received 16 January 2011; accepted 29 April 2011; published online 15 June 2011)

\begin{abstract}
We have analyzed the structural and optical properties of Si implanted with very high Ti doses and subsequently pulsed-laser melted (PLM). After PLM, all samples exhibit an abrupt and roughly uniform, box-shaped Ti profile, with a concentration around $2 \times 10^{20} \mathrm{~cm}^{-3}$, which is well above the Mott limit, within a $150 \mathrm{~nm}$ thick layer. Samples PLM-annealed at the highest energy density $\left(1.8 \mathrm{~J} / \mathrm{cm}^{2}\right)$ exhibit good lattice reconstruction. Independent of the annealing energy density, in all of the samples we observe strong sub-bandgap absorption, with absorption coefficient values between $4 \times 10^{3}$ and $10^{4} \mathrm{~cm}^{-1}$. These results are explained in terms of the formation of an intermediate band (IB) originated from the Ti deep levels. (C) 2011 American Institute of Physics. [doi:10.1063/1.3596525]
\end{abstract}

\section{INTRODUCTION}

The third generation of photovoltaic devices ${ }^{1}$ approach aims to substantially increase the efficiency of solar cells by decreasing energy losses. One of the main power loss mechanisms that limit their efficiency is the inability to absorb photons with energy lower than the bandgap. An exciting possibility for overcoming this limitation is the implementation of the so-called intermediate band solar cell (IBSC). ${ }^{2}$

The IBSC is a forward-looking approach based on the idea of placing a third band of allowed states between the valence and conduction bands. This new band would allow the absorption of photons of energy lower than the semiconductor bandgap by means of valence-to-intermediate band and intermediate-to-conduction band transitions. This mechanism would lead to strong sub-bandgap absorption, therefore increasing the photocurrent and ultimately producing a more efficient device.

If an IB material based on $\mathrm{Si}$ is obtained, it would potentially be possible to obtain $\mathrm{Si}$ solar cells with higher efficiency than the present devices, which would be extremely important, taking into account that $\mathrm{Si}$ solar cells cover most of the photovoltaic market. Additionally, an IB device based on $\mathrm{Si}$ could be attractive as a near-infrared photodetector or as a light-emitter device. ${ }^{3}$

Moreover, IB materials have attracted attention for hotcarriers solar cells, in which they could act as selective contacts to avoid the thermalization of carriers. ${ }^{1}$ Finally, IB materials could be interesting from the fundamental physics research point of view, due to their exotic properties.

Recently, it has been reported a single-junction solar cell based on ZnTe:O, in which the IB was obtained by the introduction of $\sim 10^{19} \mathrm{~cm}^{-3}$ oxygen atoms. ${ }^{4}$ This device exhibits a substantial increase of both the short circuit current and the spectral response as compared with an identical device in which ZnTe was not oxygen-doped. The improvement in cell response was related to the sub-bandgap absorption processes

\footnotetext{
a) Author to whom correspondence should be addressed. Electronic mail: dpastor@fis.ucm.es.
}

that take place between the valence, intermediate, and conduction bands. The IB formation in $\mathrm{ZnTe}: \mathrm{O}$ was related to the oxygen doping, because of this approach is simpler than those used to build up more complex devices based on a multijunction structure. ${ }^{5}$

One way to obtain an IB material based on Si is by introducing a concentration of deep level impurities above the Mott limit in the host lattice, ${ }^{6,7}$ and therefore, orders of magnitude above the solid solubility limit. To attain this goal, nonequilibrium fabrication procedures must be used, with ion implantation and pulsed-laser melting (PLM) being the best choices at present. Sub-bandgap absorption in $\mathrm{Si}$ implanted with chalcogenide elements using these techniques has recently been reported. ${ }^{8,9}$

Pulsed-laser melting is a highly nonequilibrium processing technique which is able to melt and recrystallize the $\mathrm{Si}$ surface up to several hundreds of nanometers deep in a very short time $\left(10^{-8}-10^{-6} \mathrm{~s}\right) .{ }^{10}$ This rapid recrystallization time allows the incorporation of $\mathrm{Ti}$ atoms to the $\mathrm{Si}$ at concentrations well above the Mott limit for this element. ${ }^{11}$ Additionally, the PLM processing of the Ti-implanted Si layer prevents secondary phase formation (i.e., titanium silicide), even when the equilibrium solid solubility limit has been greatly exceeded.

In recent papers, we have reported the electrical ${ }^{11}$ and structural properties ${ }^{12}$ of Ti-implanted Si samples with doses above the Mott limit. These samples showed unusual electrical transport properties ${ }^{13,14}$ and the strong reduction of the nonradiative recombination mechanism. ${ }^{15}$ All of these results point to the formation of the IB material. In this paper we present further proof of the IB formation by means of the measurement of strong sub-bandgap absorption in $\mathrm{Ti}$ implanted Si samples.

\section{EXPERIMENTAL}

N-type (111) Si samples with a thickness of $300 \mu \mathrm{m}$ ( $\mu=1450 \mathrm{~cm}^{2} / \mathrm{Vs} ; \mathrm{n}=2.2 \times 10^{13} \mathrm{~cm}^{-3}$ at room temperature), were doubly ion implanted with ${ }^{48} \mathrm{Ti}^{+}$at 35 and $150 \mathrm{keV}$, with implantation doses of $10^{15} \mathrm{~cm}^{-2}$ and $4 \times 10^{15} \mathrm{~cm}^{-2}$, 
respectively, in an IBS refurbished VARIAN CF3000 ion implanter. These implantation conditions were chosen to obtain a homogeneous Ti profile. All of the samples were tilted $\sim 7^{\circ}$ with respect to the incident beam axis to avoid channeling effects. The PLM was performed with a KrF excimer laser (one pulse, $248 \mathrm{~nm}, 20 \mathrm{~ns}$ total duration) at J.P. Sercel Associates, Inc. (New Hampshire, USA). Different laser energy densities were used $\left(1.0,1.2,1.4,1.6\right.$, and $\left.1.8 \mathrm{~J} / \mathrm{cm}^{2}\right)$. These values are well below the surface damage threshold of Si that was estimated to be above $2.3 \mathrm{~J} / \mathrm{cm}^{2}$ for a similar excimer laser melting process. ${ }^{16}$

The samples were characterized with time of flight secondary ion mass spectrometry (ToF-SIMS). This was carried out with a TOF_SIMS IV model manufactured by ION-TOF, using a $25 \mathrm{keV}$ pulsed ${ }^{3} \mathrm{Bi}^{+}$beam at a $45^{\circ}$ angle of incidence. The secondary ions generated were extracted with a $10 \mathrm{keV}$ voltage and their time of flight from the sample to the detector was measured in a reflection mass spectrometer. The raw profiles were calibrated by standard procedures, i.e., the concentration values were calculated from the ToF-SIMS counts by matching the $\mathrm{Ti}$ content in the implanted, but not annealed sample to the nominal amount of Ti. The depth was calculated by optical profilometry measurement of the crater depth and by assuming a constant erosion rate.

The crystalline quality of the implanted layers was determined by cross-sectional transmission electron microscopy (XTEM) experiments at Evans Analytical Group (Sunnyvale, CA) with a JEOL 2010 TEM working at $200 \mathrm{keV}$. The XTEM samples were obtained using the in situ focused ion beam (FIB) lift out method. This method employs the local capping of the sample with protective carbon and platinum layers. A carbon layer was applied at room temperature on top of the surface of the samples to limit the interaction of the FIB ion beam with the implanted surface layers. Bright-field images were obtained. Data was acquired using low dose TEM imaging due to the possibility of e-beam sensitivity. Simultaneously with the XTEM measurements, electron diffraction (ED) patterns with a selected area of diffraction of about $50 \mathrm{~nm}$ were obtained. The ED images provided information of the crystalline morphology of the implanted layer.

The optical properties were evaluated with transmittance and reflectance measurements performed in a Perkin Elmer Lambda 9 UV-VIS-IR spectrophotometer in the $0.55-1.2 \mathrm{eV}$ range. Reflectance measurements were conducted using an integrating sphere photodetector in the same energy range.

Additionally, transmittance was also measured in the $0.05-0.45 \mathrm{eV}$ range using a Nicolet Magna-IR 750 series II FTIR spectrometer at normal incidence.

\section{RESULTS AND DISCUSSION}

Figure 1 shows the Ti depth profiles obtained from ToFSIMS measurements for PLM energy densities ranging from 1.0 to $1.8 \mathrm{~J} / \mathrm{cm}^{2}$ together with the Ti depth profile of the asimplanted Si sample. The PLM process can be described by two different stages. ${ }^{17}$ In the melting stage, a superficial layer of the sample is melted, and the impurities within this thickness tend to homogeneously spread in the melted

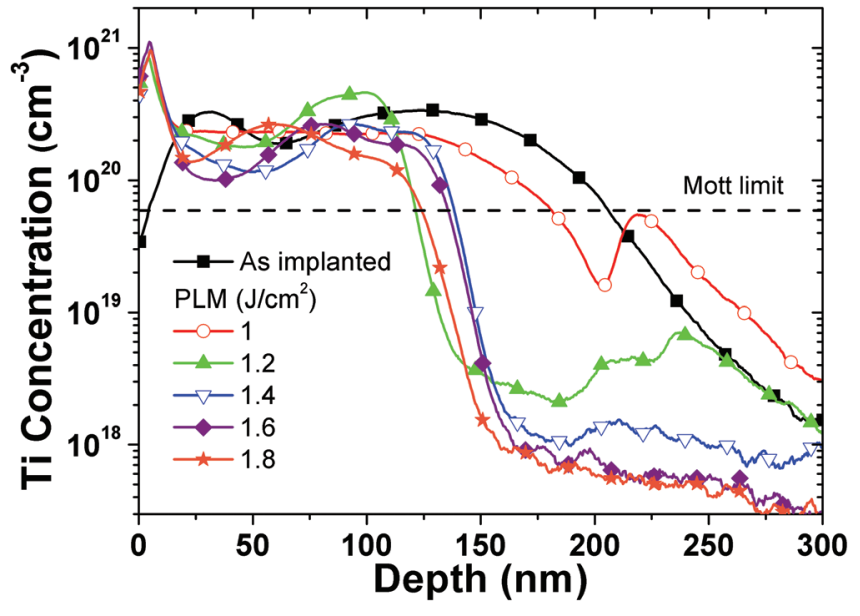

FIG. 1. (Color online) ToF-SIMS profiles of samples with double implantation $\left(35 \mathrm{keV}, 10^{15} \mathrm{~cm}^{-2} ; 150 \mathrm{keV}, 4.5 \times 10^{15} \mathrm{~cm}^{-2}\right)$ as-implanted and after different PLM processes.

region, because the diffusion coefficients and the solubility limit are orders of magnitude higher than in the solid phase. In the solidifying stage, the melted front moves toward the surface of the Si: $\mathrm{Ti}^{+}$layer. Since the solubility limit in the solid phase is orders of magnitude lower than in the melted phase, a push effect takes place, displacing the impurities to the surface of the sample. ${ }^{18}$

As shown in Fig. 1 for the as-implanted sample, the double implantation process at two different energies achieves a uniformly distributed $\mathrm{Ti}$ concentration well above the Mott limit. However, an undesirable profile with a Gaussian-like tail in the deeper region is observed. Conversely, after PLM, a box-shaped profile of impurities is obtained, with a very sharp and abrupt tail. This is an interesting result for IB materials, since the impurity concentration below the Mott limit could deteriorate the solar cell performance by decreasing the lifetime of the carriers and increasing the nonradiative recombination. During the PLM process the thickness of the implanted zone tends to decrease because of the pushing effect. This effect is clearly observed for the higher PLM energy densities analyzed, for which a lower Ti content is obtained. This is due to the Ti evaporation from the surface produced by the high temperatures reached with the PLM energy densities employed. This evaporation effect with the energy density of the PLM has been reported in a similar transition metal. ${ }^{19}$ From the results of Fig. 1, we conclude that a uniform box-shaped impurity profile of about $150 \mathrm{~nm}$ thick is obtained for the higher PLM energy densities. The Ti concentration is roughly $2 \times 10^{20} \mathrm{~cm}^{-3}$, i.e. well above the Mott limit. ${ }^{7}$

In regard to the structural characterization of samples, Figs. 2(a) and 2(b) show the XTEM images of samples PLM annealed at 1.0 and $1.8 \mathrm{~J} / \mathrm{cm}^{2}$, respectively. In both figures, we have included insets with the ED diagrams of the implanted zone and the Si substrate. The XTEM image of Fig. 2(a) shows a very defective Ti-implanted layer about $220 \mathrm{~nm}$ thick and also a defective transition zone about $50 \mathrm{~nm}$ thick. A columnar structure at the superficial layer clearly indicates that the PLM process for this sample was not sufficient to fully recover the crystallinity of the implanted zone. 

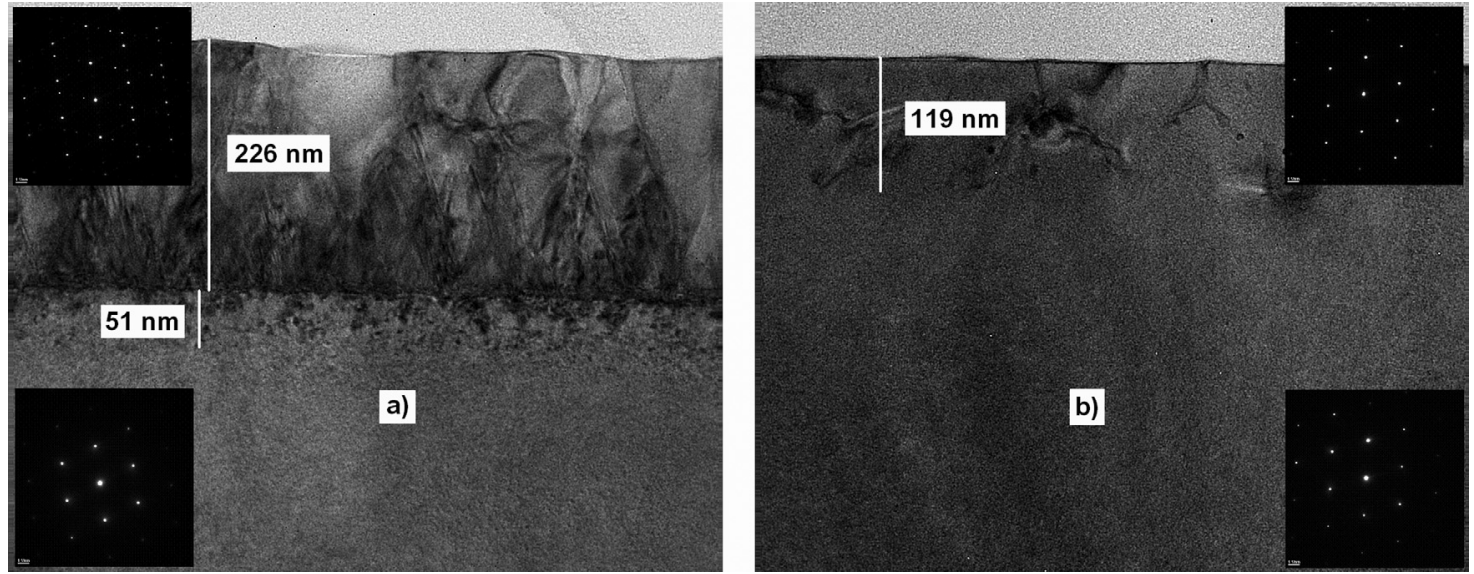

FIG. 2. (a) XTEM image of a sample PLM annealed at $1 \mathrm{~J} / \mathrm{cm}^{2}$. (b) XTEM image of a sample PLM annealed at $1.8 \mathrm{~J} / \mathrm{cm}^{2}$. The ED diagrams of both the implanted zone and the substrate are included.

Moreover, the valley in the ToF-SIMS profile of the sample PLM annealed at $1.0 \mathrm{~J} / \mathrm{cm}^{2}$ indicates the depth of the melt, and it is related to the interface found at $220 \mathrm{~nm}$ in the XTEM image. This depth is not enough to melt the whole implanted layer, and during the solidifying stage the sample recrystallizes from the seed of a damaged region and hence the crystallinity recovery is poor. Consequently, the ED diagram of the superficial layer in Fig. 2(a) presents an unusual pattern: the bright spots correspond to the (111) zone axes of the Si, but intermediate spots appear between the main dots. This effect has been associated with the highly defective lattice reconstruction. $^{12,20}$

Figure 2(b) presents the XTEM image of a sample PLM annealed at $1.8 \mathrm{~J} / \mathrm{cm}^{2}$. An overall good lattice recovery takes place and, in some zones, this recovery reaches the surface of the sample, though some defective areas are still present. The analysis of the ED patterns of both the implanted zone and the substrate did not show any differences. This result points to a good lattice reconstruction, especially compared to the sample PLM annealed at $1.0 \mathrm{~J} / \mathrm{cm}^{2}$. We have recently investigated Ti-implanted Si samples with similar doses and we have not observed silicide phase formation. ${ }^{12}$ This sili-

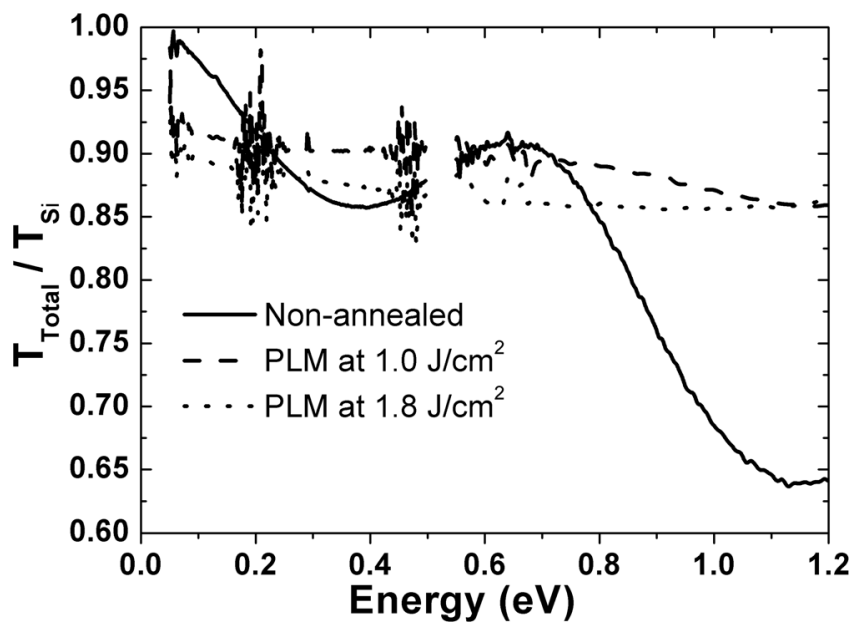

FIG. 3. Transmittance relative to $\mathrm{Si}$ substrate transmittance for the nonannealed sample and samples annealed at 1.0 and $1.8 \mathrm{~J} / \mathrm{cm}^{2}$. cide phase formation has been detected only for much higher implantation doses. ${ }^{21}$

Concerning the optical properties, Fig. 3 shows the measured transmittance $\left(T_{\text {Total }}\right)$ of the as-implanted sample and samples PLM-annealed at 1.0 and $1.8 \mathrm{~J} / \mathrm{cm}^{2}$. These results are shown relative to the transmittance of a $\mathrm{Si}$ substrate $\left(T_{\mathrm{Si}}\right)$ measured with the same conditions. Similar results were obtained for samples annealed at intermediate energy densities (which we have not shown for the sake of clarity). The features observed around 0.2 and $0.47 \mathrm{eV}$ are related to water absorption. A significant decrease of transmittance is observed for the implanted samples with respect to $\mathrm{Si}$ over the whole energy range analyzed. It must be noted that the as-implanted sample exhibits a different behavior, with a much stronger decrease of transmittance for energies in the region close to the Si bandgap energy. However, for energies below $0.2 \mathrm{eV}$, the annealed samples show a higher decrease of transmittance with respect to $\mathrm{Si}$. This different behavior may be related to the amorphous structure of the as-implanted nonannealed sample.

Reflectance relative to the $\mathrm{Si}$ reflectance $\left(R_{\mathrm{Total}} / R_{\mathrm{Si}}\right)$ is shown in Fig. 4 for the as-implanted sample and samples

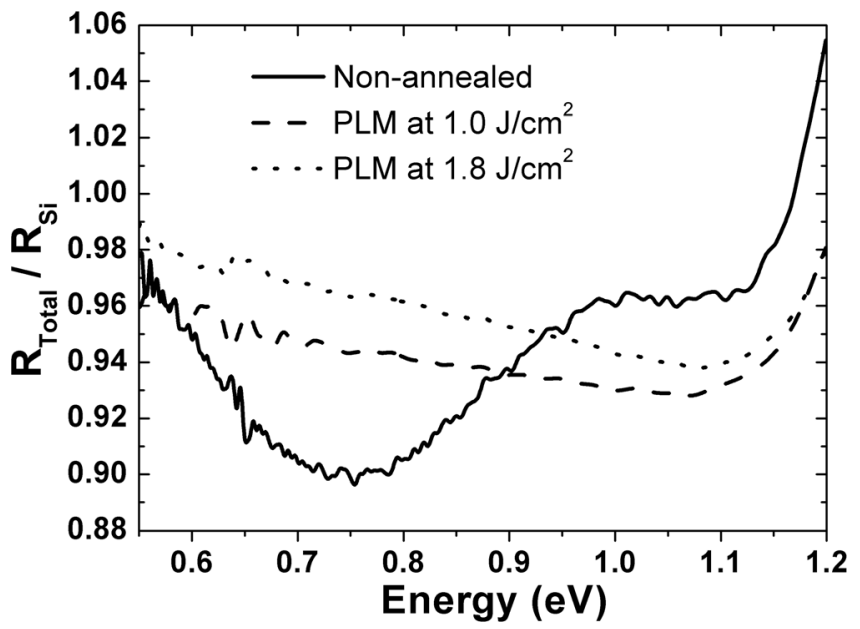

FIG. 4. Reflectance relative to $\mathrm{Si}$ substrate reflectance for the nonannealed sample and samples annealed at 1.0 and $1.8 \mathrm{~J} / \mathrm{cm}^{2}$. 
annealed at 1.0 and $1.8 \mathrm{~J} / \mathrm{cm}^{2}$. Light was incident on the implanted layer in these measurements. A decrease of reflectance for the as-implanted and PLM annealed samples with respect to $\mathrm{Si}$ is also observed. Therefore, taking into account the reduction in both transmittance and reflectance, it is clear that an increase of absorptance for energies below the fundamental bandgap transition takes place in the Ti implanted samples. Similar sub-bandgap absorption has been recently reported for chalcogen-implanted Si samples. ${ }^{8}$

In order to quantify the absorption coefficient of the implanted layers, a simple model including the interface between the implanted layer and air and the interface between the Si substrate and air has been considered. Possible reflections taking place at the interface between the implanted layer and the Si substrate have been neglected to simplify the model. This simplification will be verified later. According to this model, transmittance and reflectance of the implanted samples are given by, ${ }^{22}$

$$
\begin{gathered}
T_{\text {Total }}=\frac{\left(1-r_{i l}\right)\left(1-r_{\mathrm{Si}}\right) \exp \left(-\alpha_{i l} w_{i l}\right) \exp \left(-\alpha_{\mathrm{Si}} w_{\mathrm{Si}}\right)}{1-r_{i l} r_{\mathrm{Si}} \exp \left(-2 \alpha_{i l} w_{i l}\right) \exp \left(-2 \alpha_{\mathrm{Si}} w_{\mathrm{Si}}\right)}, \\
R_{\text {Total }}=r_{i l}+\frac{\left(1-r_{i l}\right)^{2} r_{\mathrm{Si}} \exp \left(-2 \alpha_{i l} w_{i l}\right) \exp \left(-2 \alpha_{\mathrm{Si}} w_{\mathrm{Si}}\right)}{1-r_{i l} r_{\mathrm{Si}} \exp \left(-2 \alpha_{i l} w_{i l}\right) \exp \left(-2 \alpha_{\mathrm{Si}} w_{\mathrm{Si}}\right)},
\end{gathered}
$$

where

$r_{i l}$ is the reflectance of the interface between air and the implanted layer,

$r_{\mathrm{Si}}$ is the reflectance of the interface between air and the Si substrate,

$\alpha_{i l}$ is the absorption coefficient of the implanted layer,

$\alpha_{\mathrm{Si}}$ is the absorption coefficient of the Si substrate,

$w_{i l}$ is the thickness of the implanted layer, and

$w_{\mathrm{Si}}$ is the thickness of the $\mathrm{Si}$ substrate.

Figure 5 shows a schematic of the structure. For an interface between air and a material with complex refractive index given by $n+i k$, the reflectance is given by,

$$
r=\frac{(n-1)^{2}+k^{2}}{(n+1)^{2}+k^{2}} .
$$

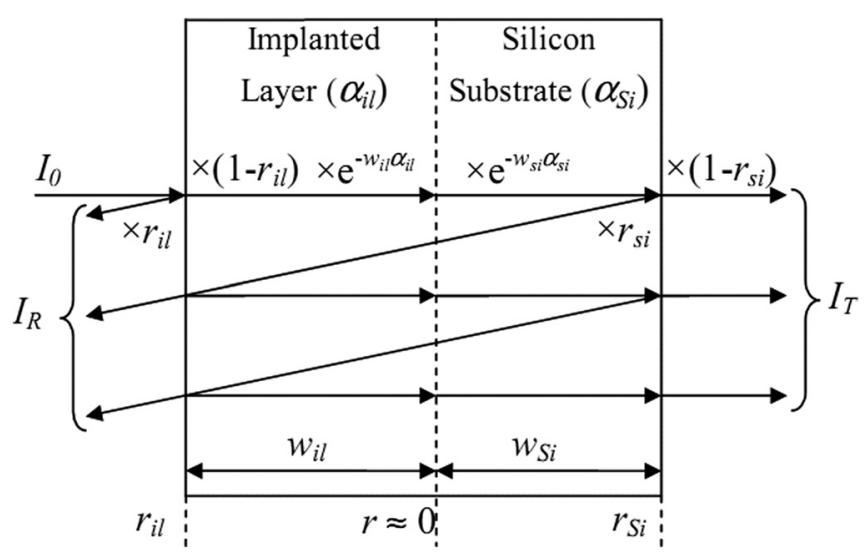

FIG. 5. Schematics of samples and parameters for calculation of the total transmittance and reflectance.

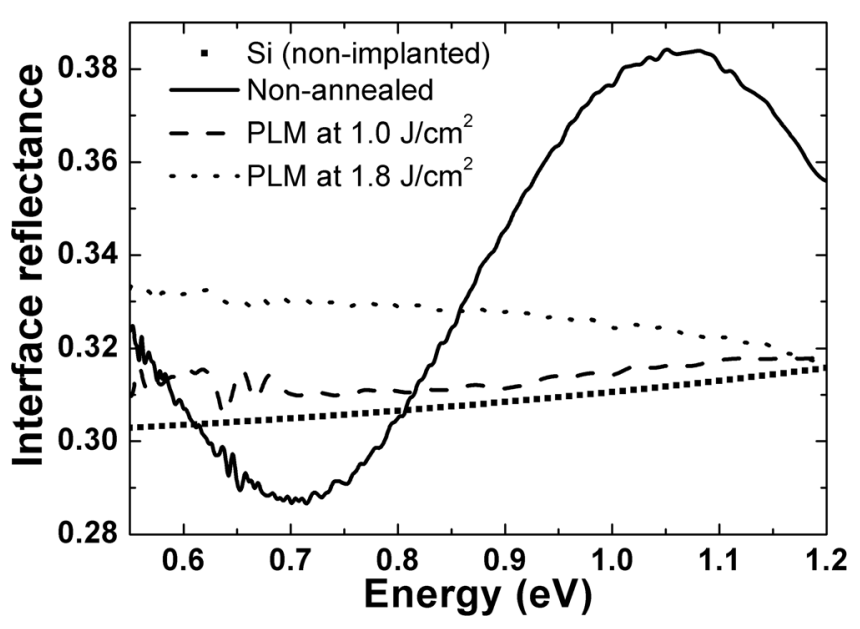

FIG. 6. Reflectance of the interface between air and the implanted layer for the nonannealed sample and samples annealed at 1.0 and $1.8 \mathrm{~J} / \mathrm{cm}^{2}$. The calculated reflectance for the air-Si interface is also shown.

For the $\mathrm{Si}$ substrate sample, the total transmittance, $T_{\mathrm{Si}}$, and the total reflectance, $R_{\mathrm{Si}}$, are calculated according to Eqs. (1) and (2), replacing $r_{i l}$ by $r_{\mathrm{Si}}$ and setting $w_{i l}=0$.

The product, $\alpha_{\mathrm{Si}} w_{\mathrm{Si}}$, was obtained from the measured $T_{\mathrm{Si}}$ using known values of the $\mathrm{Si}$ dielectric function to obtain $r_{\mathrm{Si}}{ }^{23}$ The measured reflectance of $\mathrm{Si}, R_{\mathrm{Si}}$, was not directly used in order to prevent a possible error arising from the not exact $100 \%$ reflectance of the reference sample when measuring the background. This error is compensated by using the relative reflectance $R_{\text {Total }} / R_{\mathrm{Si}}$.

Once the parameters of $\mathrm{Si}$ are known, the parameters of the implanted layer are obtained by numerically solving $T_{\mathrm{To}}$ tal $/ T_{\mathrm{Si}}$ and $R_{\text {Total }} / R_{\mathrm{Si}}$, according to Eqs. (1) and (2) for the experimental data. The results of $r_{i l}$ and $\alpha_{i l} w_{i l}$ are shown in Figs. 6 and 7, respectively, for the as-implanted sample and for samples PLM-annealed at 1.0 and $1.8 \mathrm{~J} / \mathrm{cm}^{2}$. A slight increase of the reflectance, $r_{i l}$, with respect to $r_{\mathrm{Si}}$ is observed for the implanted and PLM annealed samples. This increase has been previously predicted from theoretical calculations. ${ }^{24}$ On the contrary, the as-implanted and nonannealed sample exhibits a completely different behavior.

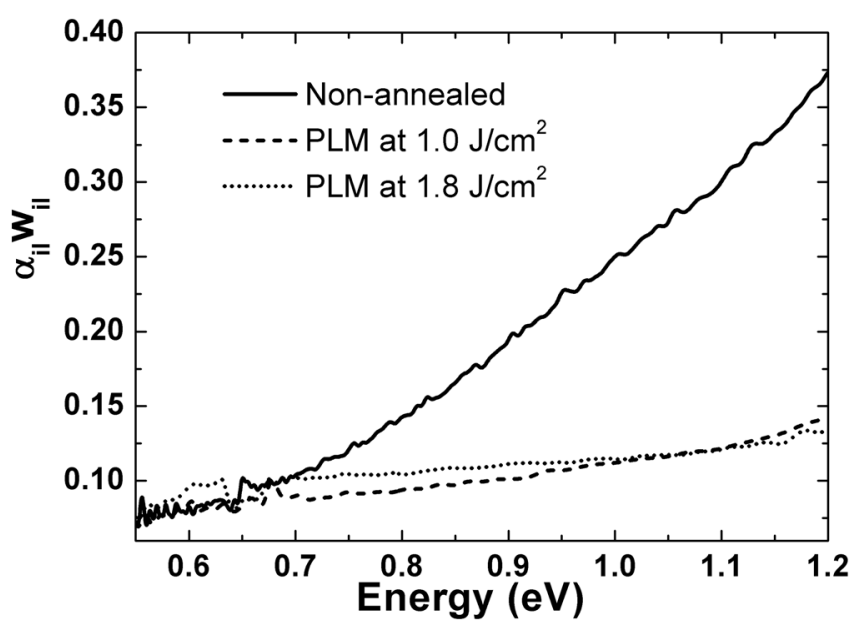

FIG. 7. Product of absorption coefficient and thickness of the implanted layer for the nonannealed sample and samples annealed at 1.0 and $1.8 \mathrm{~J} / \mathrm{cm}^{2}$. 
It must be noted that this increase in reflectance is not justified by the increase of the absorption coefficient in the implanted layer. So, the increased reflectance $r_{i l}$ with respect to $r_{\mathrm{Si}}$ may be due to changes in the real part of the implanted layer refractive index as a consequence of the Ti doping. Nevertheless, these changes are small. So, taking Eq. (3) into account, changes of the reflectance between air and the implanted layer are much more significant than changes between the implanted layer and the Si substrate, which justifies neglecting the reflectance at the implanted layer-Si interface.

Concerning the absorption, no significant differences were observed for the obtained $\alpha_{i l} w_{i l}$ values for samples annealed at different energies. However, much stronger subbandgap absorption is observed for the as-implanted sample. We attribute the different behavior of this sample to the highly defective, amorphous nature of the implanted layer, which did not undergo any lattice reconstruction process. Alternatively, for samples annealed between 1.0 and $1.8 \mathrm{~J} /$ $\mathrm{cm}^{2}$, even though a different degree of lattice recovery was achieved (as we have previously showed and discussed concerning Fig. 2), nearly the same absorption term, $\alpha_{i l} w_{i l}$, was obtained. It must be noted that lattice recovery up to the surface of the sample is achieved for samples annealed at high energy density, as shown in Fig. 2(b). Thus, an accumulation of optically active defects, such as Ti-O complexes, at the high $\mathrm{Ti}$ concentration region near the surface is unlikely. Furthermore, analysis of the oxygen profile by means of ToF-SIMS measurements performed on similar samples does not reveal any increase of the $\mathrm{O}$ concentration in this region, indicating that no optically active oxygen complexes have been formed. Therefore, we conclude that the absorption observed in the annealed samples is not related to an incomplete lattice recovery or to the presence of a high defect concentration. Otherwise, higher absorption would be observed for the more defective samples (annealed at lower energy densities). As a consequence, we assume that the measured absorption is related to the presence of a high concentration of $\mathrm{Ti}$ in the implanted layer.

In order to calculate the absorption coefficient, the thickness of the implanted layer must be taken into account. For this calculation, $w_{i l}$ was obtained from the ToF-SIMS profiles of Fig. 1, taking the values corresponding to the thickness of the layer in which the theoretical Mott limit concentration is surpassed $\left.\left(6 \times 10^{19} \mathrm{~cm}^{-3}\right)\right)^{7}$ The calculated absorption coefficient, $\alpha_{i l}$, corresponding to the implanted layer is shown in Fig. 8, for the as-implanted sample and samples annealed at $1.0 \mathrm{~J} / \mathrm{cm}^{2}, 1.4 \mathrm{~J} / \mathrm{cm}^{2}$, and $1.8 \mathrm{~J} / \mathrm{cm}^{2}$. The results for 1.2 and $1.6 \mathrm{~J} / \mathrm{cm}^{2}$ are almost identical to the results obtained for $1.4 \mathrm{~J} / \mathrm{cm}^{2}$. High values of the absorption coefficient $\left(\alpha_{i l}\right)$ in the $4 \times 10^{3} \mathrm{~cm}^{-1}-10^{4} \mathrm{~cm}^{-1}$ range were obtained. It should be noted that in this energy range, bare silicon exhibits no absorption. Furthermore, it is observed that $\alpha_{i l}$ increases as a function of energy. In the following paragraphs, we will give an explanation for this behavior.

In previous works, we demonstrated that the implanted layer is characterized by a high conductivity as a result of a high carrier concentration in the IB. ${ }^{11,13}$ Absorption associated with the free carriers can be calculated, assuming that the imaginary part of the dielectric function $\left(\varepsilon_{2}\right)$ is given by, ${ }^{25}$

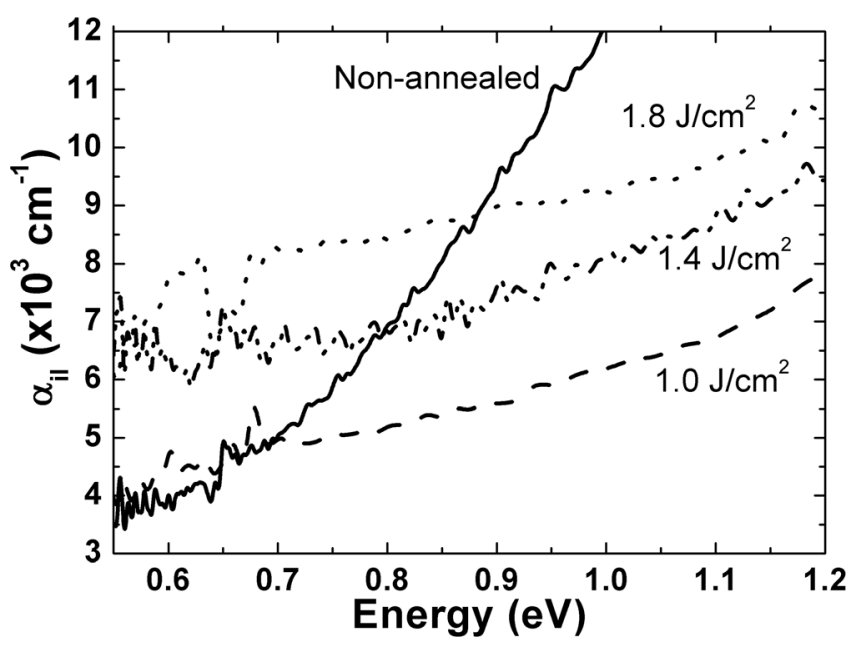

FIG. 8. Absorption coefficient of the implanted layer for the nonannealed sample and samples annealed at $1.0,1.4$, and $1.8 \mathrm{~J} / \mathrm{cm}^{2}$.

$$
\varepsilon_{2}=\frac{\sigma}{\omega \varepsilon_{0}}
$$

where $\sigma$ is the conductivity of the implanted layer, $\omega$ is the frequency, and $\epsilon_{0}$ is the permittivity of vacuum. Conductivity depends upon frequency according to, ${ }^{25}$

$$
\sigma=\frac{\sigma_{0}}{1+\omega^{2}\langle\tau\rangle^{2}}
$$

where $\langle\tau\rangle$ is the average time between carrier collisions.

From the resistivity measurements in samples with similar Ti concentrations, according to SIMS profiles, ${ }^{12}$ the conductivity of the implanted layer is estimated to be about $\sigma_{0}$ $\approx 20 \Omega^{-1} \mathrm{~cm}^{-1}$. Assuming this $\sigma_{0}$ value over the whole energy range, a roughly constant value for $\alpha_{i l}$ around $2 \times 10^{3}$ $\mathrm{cm}^{-1}$ is obtained for a hypothetical free carrier absorption mechanism, which is significantly lower than the experimental values. Furthermore, for the energy range studied $\left(\omega>8 \times 10^{14} \mathrm{rad} \mathrm{s}^{-1}\right)$, according to Eq. (5), a decrease of $\sigma$ with increasing energy is expected. This would result in orders of magnitude lower values of the absorption coefficient $\alpha_{i l}$ (depending on the exact value of $\langle\tau\rangle$ ), and to a decreasing behavior of $\alpha_{i l}$ as a function of energy. For $\omega\langle\tau\rangle$ $\gg 1, \alpha_{i 1}$ would be inversely proportional to $\omega^{2}$ (or directly proportional to $\left.\lambda^{2}\right) .^{25}$ This behavior has been widely reported for free carrier absorption in $\mathrm{Si}^{26-28}$ and it is the opposite of the one obtained for our samples. Therefore, it is concluded that the observed absorption cannot be explained by free carrier absorption and therefore, a different absorption mechanism must be taking place.

For energies below $0.55 \mathrm{eV}$, it was not possible to calculate $\alpha_{i l}$, yet the behavior of transmittance shown in Fig. 3 for annealed samples, suggests that the same absorption mechanism would be taking place at lower energies down to $0.05 \mathrm{eV}$.

Deep centers related with the presence of $\mathrm{Ti}$ in $\mathrm{Si}$ are located at different energies. In the Refs. 29 and 30, the commonly accepted values are $\mathrm{E}_{\mathrm{C}}-0.22 \mathrm{eV}, \mathrm{E}_{\mathrm{C}}-0.3 \mathrm{eV}$, and $\mathrm{E}_{\mathrm{v}}+0.29 \mathrm{eV}$. The results of Figs. 7 and 8 do not exhibit sharp features at these energies. Thus, the absorption mechanism 
present in our samples may not be attributed to impurity-level transitions. Recently, strong sub-bandgap absorption in Si samples that were heavily ion- implanted with chalcogen elements and PLM treated was reported. ${ }^{8}$ In that work, the absence of sharp features in the spectra below the bandgap was proposed to be due to the formation of an impurity band coming from the chalcogen levels. ${ }^{8}$

In Ti-implanted Si samples, we have measured unusual electronic transport properties that we only can fully explain after assuming an IB formation coming from the Ti levels introduced by ion implantation. ${ }^{31}$ Additionally, from lifetime measurements we observe that these samples exhibit a significant strong reduction of nonradiative recombination, which is also explained by the presence of an IB. ${ }^{15}$ All of this experimental proof supports the fact that the strong sub-bandgap absorption described here might be due to the presence of a Ti IB. This interesting result could lead to the definition of a completely new class of materials based on Si. These IB-Si materials could have important applications in the microelectronics field.

\section{CONCLUSIONS}

Single-crystal $\mathrm{Si}$, implanted with $\mathrm{Ti}$ at concentrations above the Mott limit and subsequently PLM annealed, exhibits strong sub-bandgap absorption. This absorption cannot be explained in terms of conventional absorption processes, such as defects or free carrier absorption. Samples with different degrees of crystalline recovery present similar values of the absorption coefficient, with an energy dependence different from the free carrier processes. We suggest that the results might be due to the formation of an IB due to the overlapping of the wave functions of $\mathrm{Ti}$ atoms located at the Si host lattice. This result, together with previously reported data on electrical transport properties and lifetime measurements reinforces the hypothesis of IB formation in these samples. In conclusion, Ti-implanted $\mathrm{Si}$ and subsequently PLM-annealed could be an interesting material for IBSC and for IB devices.

\section{ACKNOWLEDGMENTS}

The authors would like to acknowledge the Nanotechnology and Surface Analysis Services of the Universidad de Vigo C.A.C.T.I. for ToF-SIMS measurements, C.A.I.s de Técnicas Físicas and Espectroscopía of the Universidad Complutense de Madrid for ion implantation experiments and FTIR measurement facilities and Dr. J. Herrero (CIEMAT) for UV-VIS-IR measurements facilities.

This work was partially supported by the Projects GENESIS-FV (Grant No. CSD2006-0004) funded by the Spanish Consolider National Programme, NUMANCIA II (Grant No.
S-2009/ENE-1477) funded by the Regional Government of Comunidad de Madrid.

${ }^{1}$ G. Conibeer, Mater. Today 10, 42 (2007).

${ }^{2}$ A. Luque and A. Martí, Phys. Rev. Lett. 78, 5014 (1997).

${ }^{3}$ C. H. Crouch, J. E. Carey, J. M. Warrender, M. J. Aziz, and E. Mazur, Appl. Phys. Lett. 84, 1850 (2004).

${ }^{4}$ Weiming Wang, Albert S. Lin, and J. D. Phillips, Appl. Phys. Lett. 95, 011103 (2009).

${ }^{5}$ J. F. Geisz, D. J. Friedman, J. S. Ward, A. Duda, W. J. Olavarria, T. E. Moriarty, J. T. Kiehl, M. J. Romero, A. G. Norman, and K. M. Jones, Appl. Phys. Lett. 93, 123505 (2008).

${ }^{6}$ N. F. Mott, Rev. Mod. Phys. 40, 677 (1968).

${ }^{7}$ A. Luque, A. Martí, E. Antolín, and C. Tablero, Physica B 382, 320 (2006).

${ }^{8}$ T. G. Kim, J. M. Warrender, and M. J. Aziz, Appl. Phys. Lett. 88, 241902 (2006).

${ }^{9}$ P. B. Brion, A. Kohno, S. Charnvanichborikarn, J. M. Warrender, I. Umezu, M. Tabbal, J. S. Williams, and M. J. Aziz, J. Appl. Phys. 107, 123506 (2010).

${ }^{10}$ J. Narayan, C.W. White, M. J. Aziz, B. Stritzker, and A. Walthuis, J. Appl. Phys. 57, 564 (1985).

${ }^{11}$ J. Olea, M. Toledano-Luque, D. Pastor, I. Mártil, and G. González-Díaz, J. Appl. Phys. 104, 016105 (2008).

${ }^{12}$ J. Olea, M. Toledano-Luque, D. Pastor, E. San-Andrés, I. Mártil, and G. González-Díaz, J. Appl. Phys. 107, 103524 (2010).

${ }^{13}$ J. Olea, G. González-Díaz, D. Pastor, and I. Mártil, J. Phys. D: Appl. Phys. 42, 085110 (2009).

${ }^{14}$ J. Olea, D. Pastor, I. Mártil, and G. González-Díaz, Sol. Energy Mater. Sol. Cells 94, 1907 (2010).

${ }^{15}$ E. Antolín, A. Martí, J. Olea, D. Pastor, G. González-Díaz, I. Mártil, and A. Luque, Appl. Phys. Lett. 94, 042115 (2009).

${ }^{16}$ M. Tabbal, T. Kim, D. N. Woolf, B. Shin, and M. J. Aziz, Appl. Phys. A 98, 589 (2010).

${ }^{17}$ K. M. Yu, W. Walukiewicz, M. A. Scarpulla, O. D. Dubon, J. Wu, J. Jasinski, Z. Liliental-Weber, J. W. Beeman, M. R. Pillai, and M. J. Aziz, J. Appl. Phys. 94, 1043 (2003).

${ }^{18}$ C. W. White, S. R. Wilson, B. R. Appleton, and F. W. Young, Jr., J. Appl. Phys. 51, 738 (1980).

${ }^{19}$ B. Cui, L. Wu, and S. Y. Chou, Nanotechnology 19, 345303 (2008).

${ }^{20}$ D. B. Williams and B. C. Carter, Transmission Electron Microscopy II, Diffraction (Plenum, New York, 1996).

${ }^{21}$ J. Olea, D. Pastor, I. Mártil, G. González, J. Ibáñez, R. Cuscó, and L. Artús, MRS Fall Meeting 1210, Q04-10 (2009).

${ }^{22}$ S. M. Sze and K. K. Ng, Physics of Semiconductor Devices (John Wiley \& Sons, Hoboken, 2007).

${ }^{23}$ C. M. Herzinger, B. Johs, W. A. McGahan, J. A. Woollam, and W. Paulson, J. Appl. Phys. 83, 3323 (1998).

${ }^{24}$ K. Sanchez, I. Aguilera, V. Palacios, and P. Wahnón, Proceedings of the 24th European Photovoltaic Solar Energy Conference, 21-25 September 2009 (Hamburg, Germany, 2009).

${ }^{25}$ R. H. Bube, Electronic Properties of Crystalline Solids (Academic, New York, 1974).

${ }^{26}$ W. Spitzer and H. Y. Fan, Phys. Rev. 108, 268 (1957).

${ }^{27}$ H. Hara and Y. Nishi, J. Phys. Soc. Jpn. 21, 1222 (1966).

${ }^{28}$ D. K. Schroder, R. N. Thomos, and J. C. Swartz, IEEE Trans. Electron. Dev. ED-25, 254 (1978).

${ }^{29}$ D. Mathiot and S. Hocine, J. Appl. Phys. 66, 5862 (1989).

${ }^{30}$ J.-W. Chen, A. G. Milnes, and A. Rohatgi, Solid-State Electron. 22, 801 (1979).

${ }^{31}$ G. Gonzalez-Díaz, J. Olea, I.Mártil, D. Pastor, A. Martí, E. Antolín, and A. Luque, Sol. Energy Mater. Sol. Cells 93, 1668 (2009). 